\title{
A comparison of Adomian decomposition method and RK4 algorithm on Volterra integro differential equations of 2 nd kind
}

\author{
Kekana M.C *, Shatalov M.Y, Moshokoa S.P \\ Tshwane University of Technology, Private Bag X680, Pretoria, 0001, South Africa \\ *Corresponding author E-mail: kekanamc@tut.ac.za
}

\begin{abstract}
Copyright (C)2015 Kekana M.C et. al. This is an open access article distributed under the Creative Commons Attribution License, which permits unrestricted use, distribution, and reproduction in any medium, provided the original work is properly cited.
\end{abstract}

\begin{abstract}
In this paper,Volterra Integro differential equations are solved using the Adomian decomposition method.The solutions are obtained in form of infinite series and compared to Runge-Kutta4 algorithm. The technique is described and illustrated with examples; numerical results are also presented graphically. The software used in this paper is mathematica 10.
\end{abstract}

Keywords: Volterra Integro differential equations, Adomian decomposition method, Adomian polynomial,Runge Kutta4, Absolute error

\section{Introduction}

The analytical solutions of integro Volterra differential equations have a major role to in areas of physical science and engineering. The application of integro Volterra differential equations is in theory of electromagnetic theory, electrical circuit problems and heat and mass diffusion processes.

Adomian decomposition method (ADM) is a semi analytical method of solving both initial valued linear and nonlinear integral equation, this method generally decompose a integral equation into two parts the linear and non-linear part. The method was developed by G.Adomian[1, 2, 3, 4]. ADM has been applied successfully to solve many nonlinear differential or integrals equations, ordinary or partial with approximations which converge rapidly to the desired solution. One of the main advantages of using the ADM it has proven to be a competitive alternative method to Taylor series method. Adomian decomposition method is a useful in non-linear integrals equation and their accuracy in determined by the number of the partial solution used. ADM has significant advantages over other numerical methods

Different results has being archived while comparing the ADM with other perturbation methods. Edward et al.[5] campared ADM to Runge-Kutta on prey-predator model. Rach [7] showed the advantages of ADM over Picard iteration.El-Sayed et al.[6] compared wavelet-Galerkin method and Adomian decomposition method on integro differential equations.

Our motivation to this study is to obtain the infinite series solutions of linear and non Volterra integro differential equations using the Adomian Decomposition method and compare the results with Fourth order Runge-Kutta 
method.

\section{Analysis of Adomian decomposition method}

Let general form of Volterra integro differential eqauation

$u^{(n)}(x)=f(x)+\int_{0}^{x} K(x, t) u^{n}(t) d t$

with initials conditions $u(0), u^{\prime}(0), \ldots \ldots ., u^{n-1}(0)$ for determining the solution of, $u(x)$. Applying the inverse operator $L^{-1}$ then equation (1) becames

$L^{-1}\left(u^{(n)}(x)\right)=L^{-1} f(x)+L^{-1}\left[\int_{0}^{x} K(x, t) u^{n}(t) d t\right]$

where $L^{-1}$ would represent integration and with given initial or boundary conditions, $L^{-1}\left(u^{(n)}(x)\right)$ will give an equation for $u$ incorporating these conditions resulting in

$u(x)=g(x)+L^{-1}\left[\int_{0}^{x} K(x, t) u^{n}(t) d t\right]$

where $g(x)$ represent the function generated for the integrating $f(x)$ and using the initial/boundary conditions Assuming that the unknown functions can be represented as an infinite series

$u(x)=\sum_{0}^{\infty} u_{n}$

we set $u_{0}=g(x)$ and the remaining terms are determined by the recursive relationship. This is done by the decomposing the non-linear terms into a series of Adomian polynomial, $A_{n}$.

The non-linear term is written as $u^{n}=\sum_{n=0}^{\infty} u_{n}$.

To determine the Adomian polynomial, a parameter, $\lambda$, is introduced

$A_{n}=\frac{1}{n !} \frac{d^{n}}{d \lambda^{n}} N\left[\sum_{n=0}^{\infty} \lambda^{n} u_{n}\right]_{\lambda=0}$

then

$\sum_{n=0}^{\infty} u_{n}=g(x)+L^{-1}\left[\int_{0}^{x} K(x, t) \sum_{n=0}^{\infty} A_{n} d t\right]$ 


\section{Main results}

Example 3.1 Consider the second order linear Volterra integro differential equation

$u^{\prime \prime}(x)=x-\frac{x^{3}}{6}+\int_{0}^{x}(x-t) u(t) d t$

with initial conditions $u(0)=0$ and $u^{\prime}(0)=2$.

the algorithm of Adomian decomposition method on equations(7) it leads to the following recursive iterative formula

$$
\begin{aligned}
& u_{n+1}(x)=\frac{1}{3 !} \int_{0}^{x}(x-t)^{3} u_{n} d t \\
& \text { where } u_{0}=2 x+\frac{x^{3}}{6}-\frac{x^{5}}{120} \\
& u_{1}=\frac{x^{5}}{60}+\frac{x^{7}}{5040}-\frac{x^{9}}{362800} \\
& u_{2}=\frac{x^{9}}{181440}+\frac{x^{11}}{39916800}-\frac{x^{13}}{6227020800} \\
& u_{3}=\frac{x^{13}}{3113510400}+\frac{x^{15}}{1307674368000}-\frac{x^{17}}{355687428096000} \\
& \cdot \\
& \cdot \\
& u(x)=2 x+\frac{x^{3}}{6}+\frac{x^{5}}{120}+\frac{x^{7}}{5040}+\frac{x^{9}}{362880}+\frac{x^{11}}{39916800}+\frac{x^{13}}{6227020800}+\frac{x^{15}}{1307674368000}+\ldots \\
& u(x)=x+\left(x+\frac{x^{3}}{6}+\frac{x^{5}}{120}+\frac{x^{7}}{5040}+\frac{x^{9}}{362880}+\frac{x^{11}}{39916800}+\frac{x^{13}}{6227020800}+\frac{x^{15}}{1307674368000}+\ldots\right) \\
& u(x)=x+\sinh (x)
\end{aligned}
$$

Example 3.2 Consider the linear third order Volterra integro differential equation

$u^{\prime \prime \prime}(x)=1+x+\frac{x^{3}}{6}+\int_{0}^{x}(x-t) u(t) d t$

with initial conditions $u(0)=1, u^{\prime}(0)=0$ and $u^{\prime \prime}(0)=1$.

the algorithm of Adomian decomposition method on equations(8) it leads to the following recursive iterative formula

$$
\begin{aligned}
u_{n+1}(x) & =\frac{1}{4 !} \int_{0}^{x}(x-t)^{4} u_{n} d t \\
u_{0} & =1+\frac{x^{2}}{2}+\frac{x^{3}}{6}+\frac{x^{4}}{24}+\frac{x^{6}}{720} \\
u_{1} & =\frac{x^{5}}{120}+\frac{x^{7}}{5040}+\frac{x^{8}}{40320}+\frac{x^{11}}{39916800} \\
u_{2} & =\frac{x^{10}}{3628800}+\frac{x^{12}}{479001600}+\frac{x^{13}}{6227020800}+\frac{x^{14}}{87178291200}+\frac{x^{16}}{20922789888000} \\
u_{3} & =\frac{x^{15}}{1307674368000}+\frac{x^{17}}{355687428096000}+\frac{x^{18}}{6402373705728000}+\frac{x^{19}}{121645100408832000}+\frac{x^{21}}{51090942171709440000}
\end{aligned}
$$




$$
\begin{aligned}
& u(x)=1+\frac{x^{2}}{2}+\frac{x^{3}}{6}+\frac{x^{4}}{24}+\frac{x^{5}}{120}+\frac{x^{6}}{720}+\frac{x^{7}}{5040}+\frac{x^{8}}{40320}+\frac{x^{9}}{362880}+\frac{x^{10}}{3628800}+. . \\
& u(x)=\left(1+x+\frac{x^{2}}{2}+\frac{x^{3}}{6}+\frac{x^{4}}{24}+\frac{x^{5}}{120}+\frac{x^{6}}{720}+\frac{x^{7}}{5040}+\frac{x^{8}}{40320}+\frac{x^{9}}{362880}+. .\right)-x \\
& u(x)=e^{x}-x
\end{aligned}
$$

Example 3.3 Consider the non-linear Volterra integro differential equation

$u^{\prime}(x)=1+\int_{0}^{x} u^{2}(t) d t$

with initial condition $u(0)=0$.

The algorithm of Adomian decomposition method on equations(9) it leads to the following recursive iterative formula

$$
u_{n+1}(x)=\int_{0}^{x}(x-t) A_{n} d t
$$

where $u_{0}=x$

$A_{n}$ is the Adomian polymomial evaluated by

$$
\begin{aligned}
A_{0} & =u_{0}^{2} \\
A_{1} & =2 u_{0} u_{1} \\
A_{2} & =u_{1}^{2}+2 u_{0} u_{2} \\
A_{3} & =2 u_{1} u_{2}+2 u_{0} u_{3} \\
A_{4} & =u_{2}^{2}+2 u_{1} u_{3}+2 u_{0} u_{4} \\
A_{5} & =2 u_{2} u_{3}+2 u_{1} u_{4}+2 u_{0} u_{5} \\
u_{1} & =\frac{x^{4}}{12} \\
u_{2} & =\frac{x^{7}}{252} \\
u_{3} & =\frac{x^{10}}{6048} \\
u_{4} & =\frac{x^{13}}{157248} \\
u_{5} & =\frac{37 x^{16}}{158505984} \\
u_{6} & =\frac{25 x^{19}}{3011613696} \\
\cdot & \\
u(x) & =x+\frac{x^{4}}{12}+\frac{x^{7}}{252}+\frac{x^{10}}{6048}+\frac{x^{13}}{157248}+\frac{37 x^{16}}{158505984}+\frac{25 x^{19}}{3011613696}+\ldots \ldots \ldots
\end{aligned}
$$

\section{Figure and tables}


Table 1: Generated from Example3.1

\begin{tabular}{cccc}
\hline x-values & u-ADM & u-RK4 & Absolute Error \\
\hline 0 & 0 & 0 & 0 \\
0.1 & 0.200166769 & 0.200166750 & $2 . \times 10^{-8}$ \\
0.2 & 0.401336021 & 0.401336003 & $2 . \times 10^{-8}$ \\
0.3 & 0.604520314 & 0.604520293 & $2 . \times 10^{-8}$ \\
0.4 & 0.810752349 & 0.810752326 & $2 . \times 10^{-8}$ \\
0.5 & 1.02109534 & 1.02109531 & $3 . \times 10^{-8}$ \\
0.6 & 1.23665362 & 1.23665358 & $4 . \times 10^{-8}$ \\
0.7 & 1.45858374 & 1.45858370 & $5 . \times 10^{-8}$ \\
0.8 & 1.68810603 & 1.68810598 & $5 . \times 10^{-8}$ \\
0.9 & 1.92651678 & 1.92651673 & $5 . \times 10^{-8}$ \\
1.0 & 2.17520125 & 2.17520119 & $6 . \times 10^{-8}$ \\
\hline
\end{tabular}

Table 2: Generated from Example3.2

\begin{tabular}{cccc}
\hline x-values & u-ADM & u-RK4 & Absolute Error \\
\hline 0 & 1.000000000 & 1.000000000 & $0 . \times 10^{-10}$ \\
0.1 & 1.005170918 & 1.005170918 & $0 . \times 10^{-10}$ \\
0.2 & 1.021402758 & 1.021402758 & $0 . \times 10^{-10}$ \\
0.3 & 1.049858808 & 1.049858808 & $0 . \times 10^{-10}$ \\
0.4 & 1.091824698 & 1.091824698 & $0 . \times 10^{-10}$ \\
0.5 & 1.148721271 & 1.148721271 & $0 . \times 10^{-10}$ \\
0.6 & 1.222118800 & 1.222118800 & $0 . \times 10^{-10}$ \\
0.7 & 1.313752707 & 1.313752707 & $0 . \times 10^{-10}$ \\
0.8 & 1.425540928 & 1.425540928 & $0 . \times 10^{-10}$ \\
0.9 & 1.559603111 & 1.559603111 & $0 . \times 10^{-10}$ \\
1.0 & 1.718281828 & 1.718281828 & $0 . \times 10^{-10}$ \\
\hline
\end{tabular}

Table 3: Generated from Example3.3

\begin{tabular}{cccc}
\hline X-values & u-ADM & u-RK4 & Absolute Error \\
\hline 0 & 0 & 0 & 0 \\
0.1 & 0.10000833 & 0.10000833 & $0 . \times 10^{-9}$ \\
0.2 & 0.20013338 & 0.20013338 & $0 . \times 10^{-9}$ \\
0.3 & 0.30067587 & 0.30067587 & $0 . \times 10^{-9}$ \\
0.4 & 0.40213985 & 0.40213985 & $0 . \times 10^{-9}$ \\
0.5 & 0.50523950 & 0.50523950 & $0 . \times 10^{-8}$ \\
0.6 & 0.61091209 & 0.61091209 & $0 . \times 10^{-8}$ \\
0.7 & 0.72033987 & 0.72033987 & $0 . \times 10^{-8}$ \\
0.8 & 0.83498365 & 0.83498365 & $0 . \times 10^{-8}$ \\
0.9 & 0.95663232 & 0.95663232 & $0 . \times 10^{-8}$ \\
1.0 & 1.0874735 & 1.0874735 & $0 . \times 10^{-8}$ \\
\hline
\end{tabular}




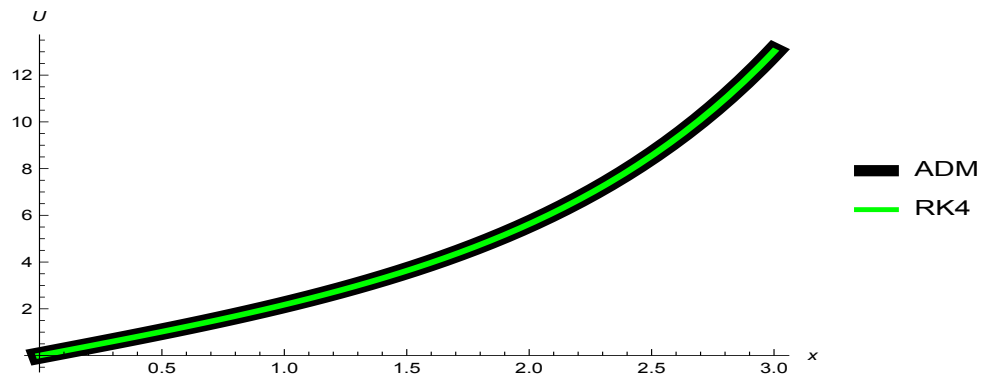

Figure 1: Generated from Example 3.1

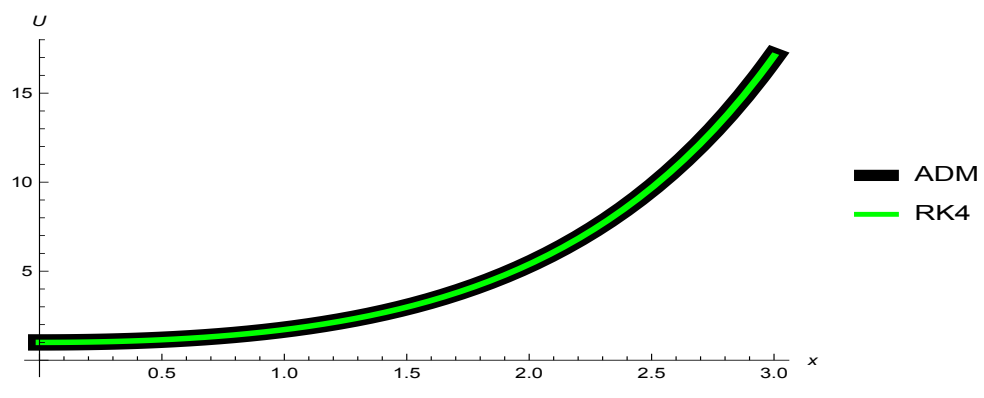

Figure 2: Generated from Example 3.2

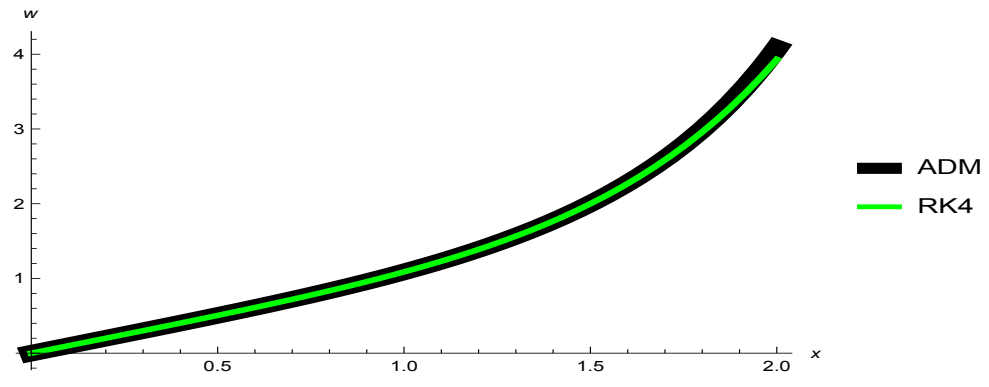

Figure 3: Generated from Example 3.3 


\section{Conclusion}

In this paper, the Adomian decomposition method for the solution of linear and non linear Volterra integro differential equations is successfully implemented. In both example3.1 and 3.2 results of the linear Volterra integro differential equations converges to the desired analytical solutions and very good accuracy is attained when comparing with Runge-Kutta4 algorithm. On Example 3.3 the non linear Volterra integro also good accuracy is attained. Adomian method can be used to solve any Volterra Integro differential equation either linear or non-linear.

\section{Acknowledgements}

The author(s) like to thank the Tshwane university of Technology for their financial support.

\section{References}

[1] G. Adomian, Solving Frontier Problems of Physics, Kluwer, Boston,1994.

[2] G. Adomian, Nonlinear Stochastic Operator Equations Frontier, Academic Press, San Diego,1986.

[3] G. Adomian, "A review of the decomposition method and some recent results for nonlinear equation", Math.Comput. Modelling, No 7, (1992), pp. 17 - 43.

[4] G. Adomian, R.Rach, "Equality of partial solutions in the decomposition method for linear or nonlinear partial defferential equations" ,Comput.Math. Appl, No.12, (1990),pp. 9 - 12.

[5] J.Y. Edwards, J.A. Roberts, N.J. Ford, "A comparision of Adomian's decomposition method and Runge-Kutta methods for approximate solution of some predator-prey model equations" ,Manchester Centre of Computational Mathematics (1997),pp. $1-17$.

[6] S.L El-Sayed, M.R Abdel-Aziz, "A comparision of Adomian decomposition method and wavelet-Galerkin method for integro-differential equations" ,Appl.Math. Comput, No.136, (2003),pp. 151 -159.

[7] R.Rach, "On the Adomian decomposition method and comparision with Picards method" ,J.Math. Appl, No.128 (1987),pp. $480-483$.

[8] A.M Wazwaz, "A new algorithm for calculating non-linear operator",Appl.Math. Comput, No.111, (2001), 32 -51'.

[9] Z.M. Odibat ,"Differential transform method for solving Volterra integral equation with separable kernels", Math. Comput. Modelling, No.48 , (2008),pp. 1144 - 1149 